\title{
Exosome complex genes mediate RNA degradation and predict survival in mantle cell lymphoma
}

\author{
WEILONG ZHANG ${ }^{1 *}$, JUNYONG ZHU ${ }^{2 *}$, XUE HE $^{3 *}$, XIAONI LIU ${ }^{4}$, JINHANG LI $^{5}$, WEI LI ${ }^{1}$, \\ PING YANG $^{1}$, JING WANG $^{1}, \mathrm{KAI} \mathrm{HU}^{1}$, XIURU ZHANG ${ }^{3},{\text { XIRU } \mathrm{LI}^{2} \text { and HONGMEI JING }}^{1}$ \\ ${ }^{1}$ Department of Hematology, Lymphoma Research Center, Peking University Third Hospital, Beijing 100191; \\ ${ }^{2}$ Department of General Surgery, Chinese People's Liberation Army General Hospital, Beijing 100853; \\ ${ }^{3}$ Department of Pathology, Beijing Tiantan Hospital Affiliated with Capital Medical University, Beijing 100050; \\ ${ }^{4}$ Department of Respiratory Medicine, The First Affiliated Hospital of Gannan Medical University, \\ Ganzhou, Guangdong 341000; ${ }^{5}$ Department of Pathology, Chinese People's \\ Liberation Army General Hospital, Beijing 100853, P.R. China
}

Received October 27, 2018; Accepted July 26, 2019

DOI: $10.3892 / \mathrm{ol} .2019 .10850$

\begin{abstract}
Exosome complex (EXOSC) genes, which encode a multi-protein intracellular complex, mediate the degradation of various types of RNA molecules. EXOSCs, also known as polymyositis/scleroderma complexes, exist in eukaryotic cells and archaea, and primarily mediate $3^{\prime}$ to $5^{\prime}$ mRNA degradation. However, how EXOSC genes are implicated in processes of B-cell immune-associated pathways and B-cell tumorigenesis remains unclear. The present bioinformatics study indicated that 6 of 10 EXOSC genes, particularly the EXO.index, were able to predict the survival of patients with mantle cell lymphoma (MCL), by analyzing gene expression profiles of 123 patients with MCL from the Gene Expression Omnibus database. The results suggested that EXOSC gene expression may be a molecular marker for MCL. Compared with the whole transcript profile, patients with MCL with a high EXO.index exhibited poorer survival and decreased RNA levels, which was also verified in a second dataset. The EXOSC genes may be associated with DNA repair and B-cell activation pathways, which may be the cause of poorer survival of patients with MCL.
\end{abstract}

Correspondence to: Dr Hongmei Jing, Department of Hematology, Lymphoma Research Center, Peking University Third Hospital, 49 North Garden Road, Haidian, Beijing 100191, P.R. China E-mail: drjinghm@163.com

Dr Xiru Li, Department of General Surgery, Chinese People's Liberation Army General Hospital, 28 Fuxing Road, Haidian District, Beijing 100853, P.R. China

E-mail: 24681i@sina.com

*Contributed equally

Key words: exosome complex genes, mantle cell lymphoma, RNA degradation, gene expression profile

\section{Introduction}

During cell differentiation and development, RNA levels are inevitably regulated in response to environmental cues. Among the different mechanisms of RNA regulation, RNA degradation is an important method of gene expression control (1). In eukaryotic cells, the processes of mRNA degradation are generally divided into the $5^{\prime}$ to $3^{\prime}$ and the $3^{\prime}$ to 5'pathways (1). In yeast, mRNA degradation also proceeds via two pathways: Rapid 5' to 3'degradation by removing the 5'-cap structure, and slower 3 ' to 5'degradation mediated by the exosome complex (2). In human cells, exosome-mediated 3 ' to 5 'degradation is the predominant pathway (3-5). The exosome complex (EXOSC), also known as the polymyositis/scleroderma complex, is a multi-protein intracellular complex capable of degrading various types of RNA. The core of the exosome is a ring to which other proteins are attached (6). Exosome component 2 (EXOSC2), as a peripheral section of exosome component 9 (EXOSC9), combines with exosome component 4 (EXOSC4) and exosome component 7 (EXOSC7) to stabilize the ring of RNase PH-domain subunits (7). Furthermore, EXOSC4, exosome component 8 (EXOSC8) and EXOSC9 bind to adenylate-uridylate-rich element-containing RNAs (8). EXOSC10 is involved in the maturation of $5.8 \mathrm{~S}$ ribosomal (r)RNA (9). In 2017, two study groups demonstrated that RNA stability regulation has an important role in hematopoietic stem and progenitor cell differentiation and T-cell homeostasis $(10,11)$.

Mantle cell lymphoma (MCL) comprises $3-10 \%$ of all non-Hodgkin B-cell lymphoma cases, as a distinct entity with a t (q13;q32) translocation event (12-18). Among patients with MCL, 60-65-year-old males represent the predominant patient type, and the majority of cases are diagnosed at the advanced stage with extranodal involvement (15). At present, various treatments are used for MCL: Chemotherapy; bone marrow transplantation; radiotherapy; immunotherapy; and targeted therapy. However, to date, no consensus regarding the optimal treatment has been reached among physicians, and rapid 


\begin{tabular}{ccccc}
\hline A Hame & Hazard.ratio & Lower & Upper & p.value \\
\hline EXOSC5 & 4.70 & 2.58 & 8.56 & $7.0 \mathrm{E}-07$ \\
EXOSC1 & 5.87 & 2.72 & 12.69 & $5.7 \mathrm{E}-06$ \\
EXOSC4 & 2.99 & 1.72 & 5.20 & $1.1 \mathrm{E}-04$ \\
EXOSC2 & 2.03 & 1.29 & 3.18 & $2.7 \mathrm{E}-03$ \\
EXOSC7 & 1.80 & 1.16 & 2.80 & $8.9 \mathrm{E}-03$ \\
EXOSC3 & 0.76 & 0.61 & 0.94 & $1.3 \mathrm{E}-02$ \\
EXOSC8 & 0.69 & 0.39 & 1.25 & $2.2 \mathrm{E}-01$ \\
EXOSC6 & 0.88 & 0.60 & 1.28 & $4.9 \mathrm{E}-01$ \\
EXOSC9 & 1.10 & 0.76 & 1.57 & $6.2 \mathrm{E}-01$ \\
EXOSC10 & 0.88 & 0.49 & 1.58 & $6.7 \mathrm{E}-01$ \\
\hline
\end{tabular}

B Gene symbol
EXOSC8
EXOSC3
EXOSC6
EXOSC10
EXOSC9
EXOSC7
EXOSC2
EXOSC4
EXOSC5
EXOSC1

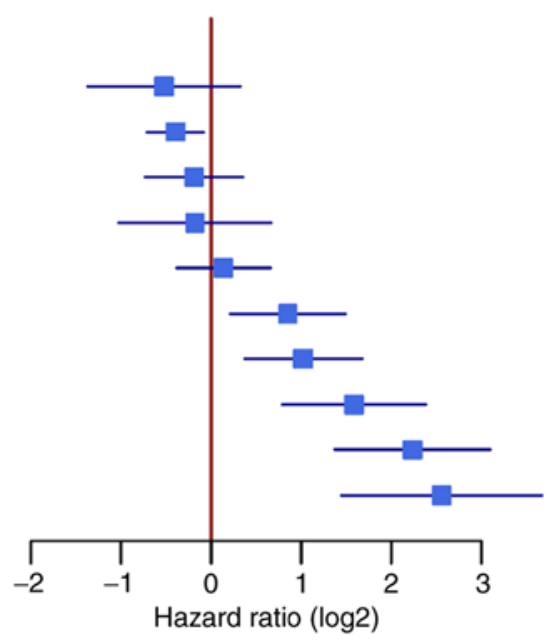

Figure 1. Survival analysis and forest plot of 10 EXOSC genes of 123 patients with mantle cell lymphoma. (A) Survival analysis of 10 EXOSC genes, classified by hazard ratio. (B) Forest plot of 10 EXOSC genes, classified by hazard ratio. The forest plot includes the lower and upper 95\% confidence intervals. EXOSC, exosome complex. EXOSC5, exosome component 5; EXOSC1, exosome component 1; EXOSC4, exosome component 4; EXOSC2, exosome component 2; EXOSC7, exosome component 7; EXOSC3, exosome component 3; EXOSC8, exosome component 8; EXOSC6, exosome component 6; EXOSC9, exosome component 9; EXOSC10, exosome component 10.

relapses hamper their curative effect (19). As a result, MCL has a relatively short median survival time of 5-7 years (20-22). To evaluate and select appropriate treatments for patients with MCL, several previous studies have investigated various approaches to stratify patients, including gene expression analysis of 20 'proliferation signature' genes (23), a PCR-based 5 -gene model (24), the antigen $\mathrm{Ki}-67$ (Ki-67) proliferation index (25) and the Mantle Cell International Prognostic Index (MIPI) (26). However, while all of these prognostic factors are associated with survival to a certain extent, none of them has been proven to be an effective tool for the selection of therapy. Therefore, it is necessary to explore the molecular mechanisms of MCL progression further, and to identify novel treatment approaches for MCL. It has been suggested that mutations in exosome component 3 (EXOSC3) may cause spinal motor neuron disease and cerebellar atrophy (27). However, little is known about the prognostic significance of EXOSC family genes in MCL. In the present study, the prognostic significance and biological implication of EXOSC genes in MCL were investigated by using a Bioinformatics analysis of gene expression data.

\section{Materials and methods}

Data sources. Affymetrix Human Genome U133 Plus 2.0 Array datasets were obtained from the NCBI Gene Expression Omnibus (GEO) database (28). The datasets GSE93291 and GSE36000 were used, containing the gene expression data of 123 and 38 MCL patients, respectively.

Gene expression analysis. The gene expression data in each probe set of all the arrays were computed using the robust multiarray averaging algorithm. Log2-transformed values were used to represent the relative RNA expression value of each probe set or gene. An unpaired Student's t-test was used to identify the differentially expressed genes. $\mathrm{P}<0.05$ was considered to indicate a statistically significant difference. Upregulated or downregulated differentially expressed genes were defined as genes with a $\log 2$ (fold change) of $>1$ or $<-1$, respectively.

Pearson's correlation coefficient was used to calculate the correlation coefficient of the expression level of 10 EXOSC genes in MCL. The 123 patients with MCL were divided into two groups using a fuzzy clustering method in 

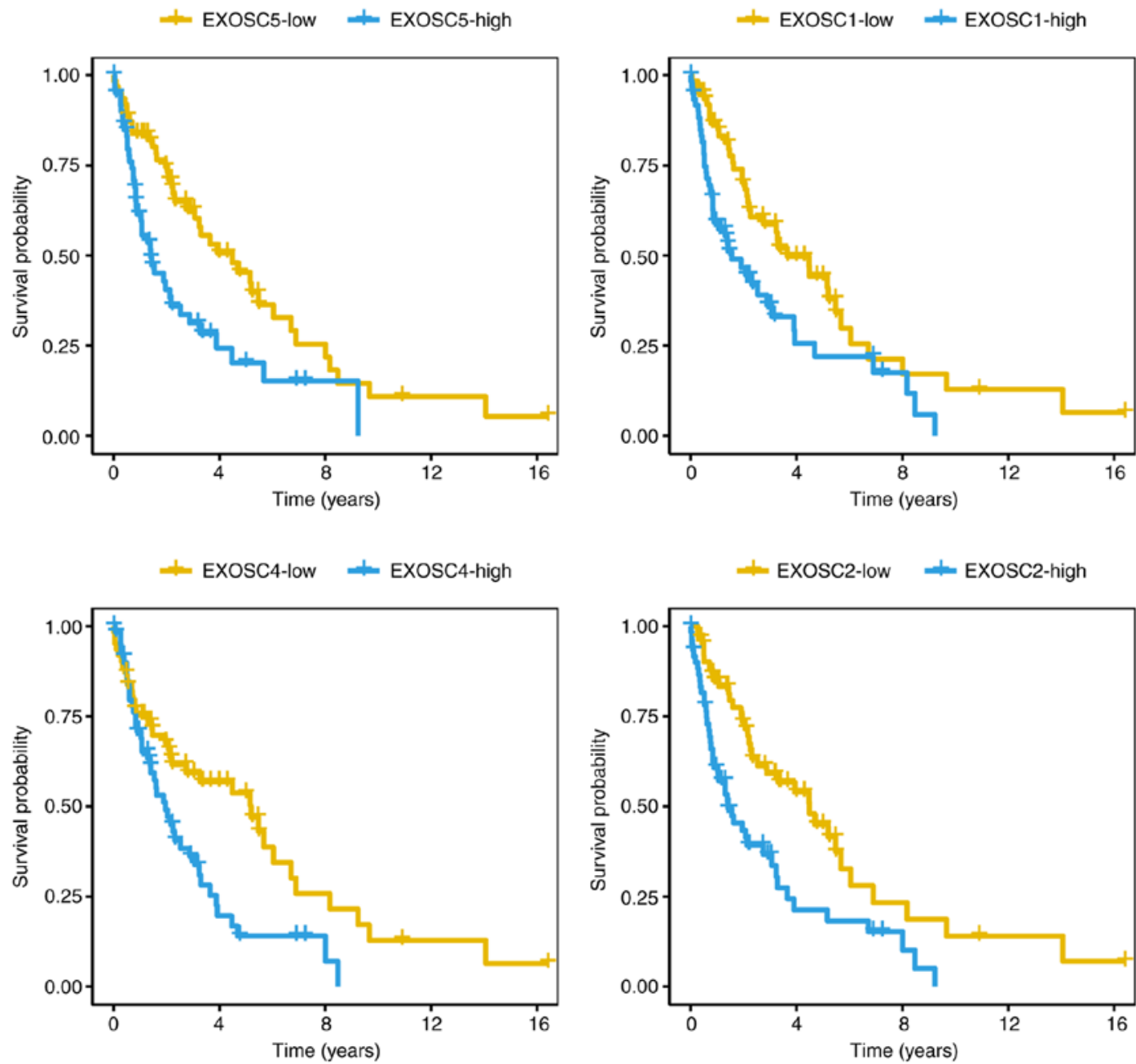

Figure 2. Kaplan-Meier curves for 4 EXOSC genes regarding overall survival of 123 patients with mantle cell lymphoma. The p-values for the EXOSC5, EXOSC1, EXOSC4 and EXOSC2 curves were as follows: $\mathrm{P}=7.0 \times 10^{-7} ; \mathrm{P}=5.7 \times 10^{-6} ; \mathrm{P}=1.1 \times 10^{-4}$; and $\mathrm{P}=2.7 \times 10^{-3}$, respectively. The log-rank test was used to compare Kaplan-Meier curves. EXOSC, exosome complex; EXOSC5, exosome component 5; EXOSC1, exosome component 1; EXOSC4, exosome component 4; EXOSC2, exosome component 2.

the ggplot2 package (version 3.1.1; ggplot2.tidyverse.org), based on the gene expression of the 10 EXOSC genes. To determine the association of EXOSC gene expression with survival of patients with MCL, Kaplan-Meier curves were calculated and log-rank tests was performed. Comparison between the expression levels of FA complementation group A (FANCA) and INO80 complex subunit D between EXO.index-high and -low groups was performed using an unpaired Student's t-test.

Definition of EXO.index for survival prediction. An EXO. index was defined to predict survival of patients with MCL. The EXO.index was calculated by using the following formula: EXO.index $\mathrm{j}_{\mathrm{j}}=\mathrm{H}_{\mathrm{j}} / \mathrm{F}_{\mathrm{j}}$, where EXO.index $\mathrm{j}_{\mathrm{j}}$ represented the index of EXOSC genes of the jth sample to predict survival. $\mathrm{H}_{\mathrm{j}}$ represented the product of the expression of harmful genes (hazard ratio of $>1$ ) with $\mathrm{P}<0.05$ in the jth sample. A total of 5 of the 10 EXOSC genes (EXOSC1, EXOSC2, EXOSC4, EXOSC5 and EXOSC7) with a hazard ratio of $>1$ were included. $F_{j}$ represented the product of the expression of favorable genes (hazard ratio of $<1$ ) of the jth sample. Of the 10 EXOSC genes, 1 gene, EXOSC 3 , with a hazard ratio of $<1$ was included.
Gene ontology (GO) analysis. The Database for Annotation and Visualization and Integrated Discovery tool was used with default parameters for GO analysis (29-31). Enriched $\mathrm{GO}$ terms $(\mathrm{P}<0.05)$ presented in the figures were manually curated, and only non-redundant GO terms in the biological process category were provided (32).

R software (version 3.1.3; www.r-project.org) was used for data analysis. Values are expressed as the mean \pm standard error of the mean in scatter plots. $\mathrm{P}<0.05$ was considered to indicate a statistically significant difference.

\section{Results}

Survival in MCL predicted by 6 of 10 EXOSC genes. To investigate the association between the EXOSC genes (EXOSC1-EXOSC10) and the survival of patients with MCL, a total of $123 \mathrm{MCL}$ expression profiles from the GEO dataset GSE93291 were analyzed. It was identified that the expression levels of 6 out of 10 EXOSC genes were significantly associated with the survival of patients with MCL $(\mathrm{P}<0.05 ; \log$-rank test). The 10 EXOSC genes were categorized based on the hazard ratio values. The genes with a hazard ratio of $<1$ were 
A

\section{EXOSC1}

$6 \quad 10$



$\bullet$

$\exists 0.42$

\section{EXOSC2}

$-0.26$

\section{$-0.22$}

$\bullet$

$\exists 0.37$



0.44

0.28

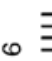

$\exists-0.06$
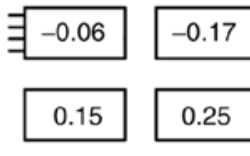

$\bullet$

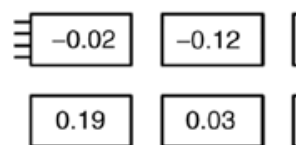

6 录



610

Tा

B
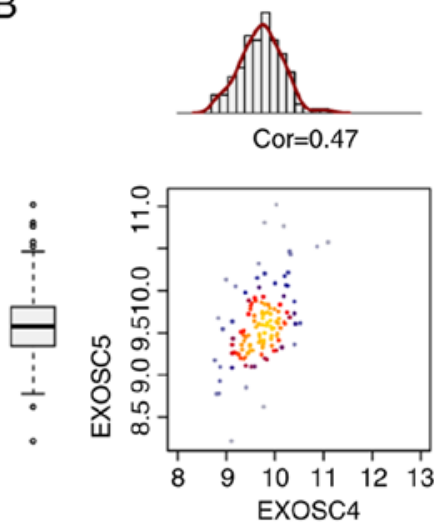

1- T-..1
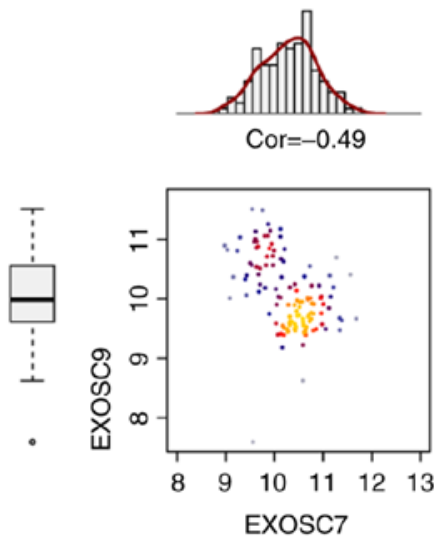

เ..-可

610
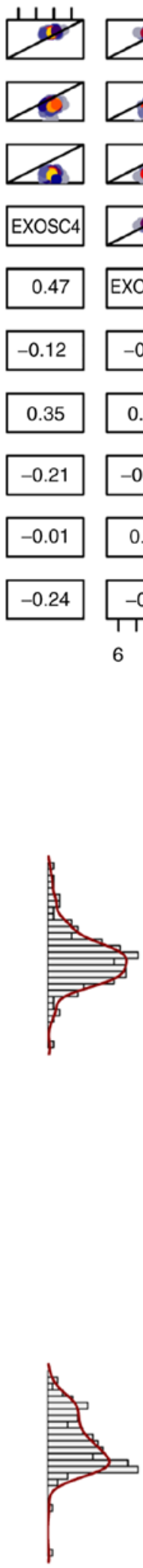
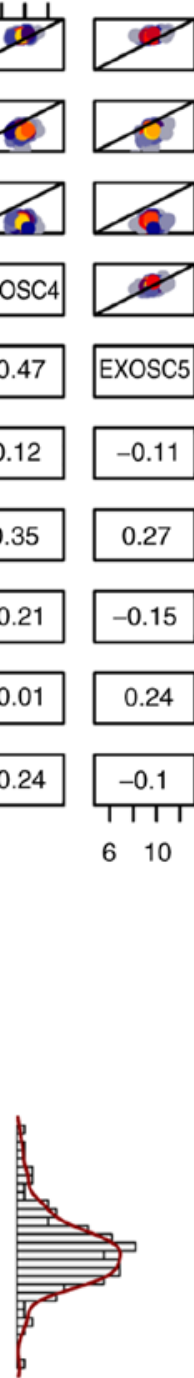

610
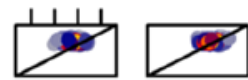

610
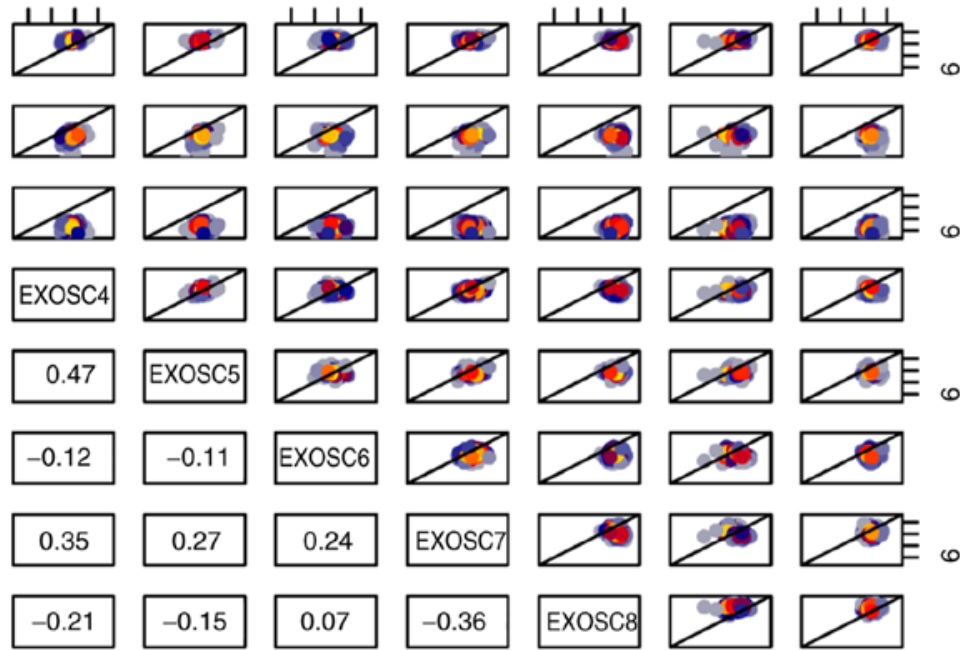

ExOSC8

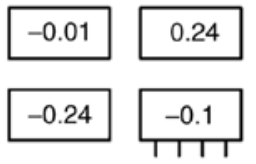

610
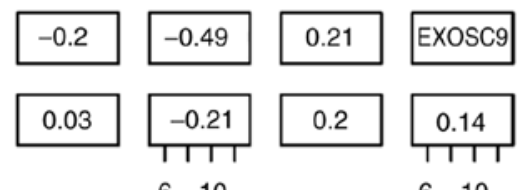

610

610
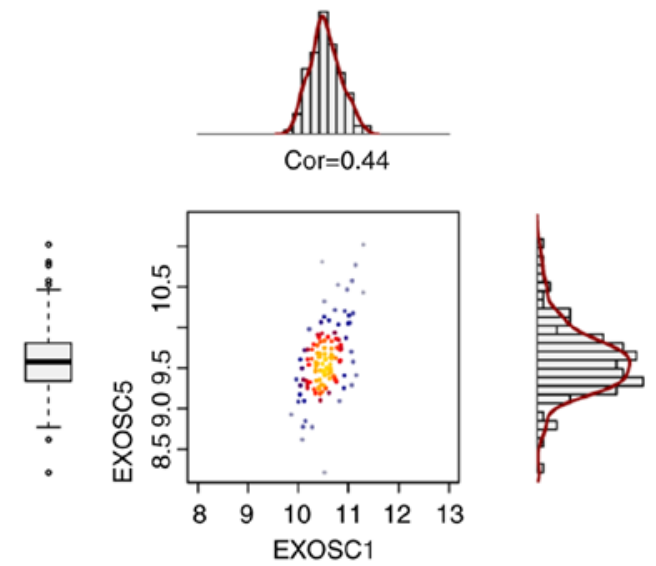

1-

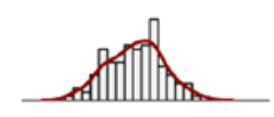

Cor $=-0.36$
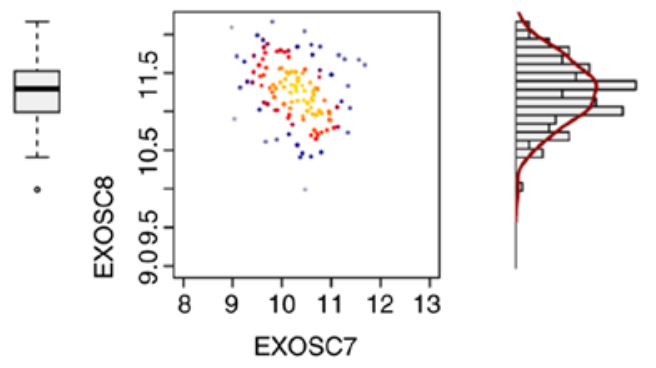

+......

Figure 3. Correlation plot of the expression levels of 10 EXOSC genes in mantle cell lymphoma. (A) Correlation plot of 10 EXOSC genes. The Pearson correlation coefficient is presented. The X-axis and Y-axis represent gene expression levels $(\log 2)$. The colors included in the upper right section of the plot represent dot density. Grey and blue represent mean low dot density and red and yellow represent high dot density. (B) Correlations between EXOSC4 and EXOSC5 (upper left), EXOSC1 and EXOSC5 (upper right), EXOSC7 and EXOSC9 (lower left), EXOSC7 and EXOSC8 (lower right) genes were calculated. EXOSC, exosome complex; cor, Pearson correlation coefficient; EXOSC4, exosome component 4; EXOSC5, exosome component 5; EXOSC1, exosome component 1; EXOSC7, exosome component 7; EXOSC9, exosome component 9; EXOSC8, exosome component 8. 
defined as 'favorable genes', as they had a positive influence on the survival of MCL. Only EXOSC3, with a hazard ratio of 0.76 [95\% confidence interval (CI), 0.61-0.94], was a significant 'favorable gene'. Conversely, the genes with a hazard ratio of $>1$ were defined as 'harmful genes' due to having a negative effect on the survival of MCL. Based on this, 5 of the 10 EXOSC genes (EXOSC1, EXOSC2, EXOSC4, EXOSC5 and EXOSC7) were classified as 'harmful genes' (Fig. 1A and B). With a hazard ratio of 5.87 (95\% CI, 2.72-12.69), EXOSC1 was the most significant gene among the 'harmful genes'. The Kaplan-Meier curves of overall survival for the 123 patients with MCL were then generated to compare 4 EXOSC genes with the log-rank test values (Fig. 2; EXOSC5, $\mathrm{P}=7.0 \times 10^{-7}$; EXOSC1, $\mathrm{P}=5.7 \times 10^{-6}$; EXOSC $4, \mathrm{P}=1.1 \times 10^{-4}$; EXOSC2, $\mathrm{P}=2.7 \times 10^{-3}$; log-rank test), indicating that the 'harmful' EXOSC genes were able to predict the survival of patients with MCL.

Correlation of the 10 EXOSC genes in MCL. Correlation plots of the expression of the 10 EXOSC genes in MCL are presented in Fig. 3. Of note, certain pairs of EXOSC genes exhibited a positive correlation, including EXOSC4 and EXOSC5 $(\mathrm{R}=0.47)$, EXOSC1 and EXOSC5 $(\mathrm{R}=0.44)$, EXOSC1 and EXOSC2 ( $\mathrm{R}=0.42)$, and EXOSC 4 and EXOSC7 $(\mathrm{R}=0.35)$. Other pairs of EXOSC genes had a negative correlation, including EXOSC7 and EXOSC9 $(\mathrm{Rr}=-0.49)$, and EXOSC7 and EXOSC8 (R=-0.36). In addition, certain pairs of EXOSC genes exhibited no correlation, including EXOSC1 and EXOSC $8(\mathrm{R}=-0.02)$, and EXOSC6 and EXOSC10 $(\mathrm{R}=-0.03)$. The 5 'harmful genes', EXOSC1, EXOSC2, EXOSC4, EXOSC5 and EXOSC7, exhibited a positive mutual correlation. EXOSC3, as a 'favorable gene', had a weak positive correlation with EXOSC8 $(\mathrm{R}=0.24)$, a weak negative correlation with EXOSC1 $(\mathrm{R}=-0.26)$ and no correlation with EXOSC5 $(\mathrm{R}=-0.08)$.

EXOSC gene expression predicts survival in MCL. Cosine correlation similarity was adopted to perform unsupervised clustering of the 10 EXOSC genes in the 123 patients with MCL (Fig. 4A). The results indicated that the 10 EXOSC genes were significantly clustered into 2 different groups (EXOSC2 and EXOSC 3 in one group, and the other 8 EXOSC genes in a second group). Furthermore, all of the 'harmful genes', with the exception of EXOSC2, were in the same group. Therefore, the EXOSC genes were considered to be associated with the survival of MCL and also exhibited a characteristic expression profile. Furthermore, using a fuzzy clustering of the 10 EXOSC genes, it was possible to stratify the 123 patients with MCL into two groups (R, ggplot2; Fig. 4B). The EXO.index was then calculated as the ratio of the expression of 'harmful genes' and 'favorable genes'. The EXO.index was significantly associated with the survival of MCL $\left(\mathrm{P}=1.73 \times 10^{-7}\right.$; log-rank test; Fig. 4C). The EXO.index revealed the unequal expression levels between the 'favorable genes' and the 'harmful genes' among the EXOSC genes. The patients with MCL in the EXO. index-high group exhibited poorer survival rates compared with the EXO.index-low group.

EXO.index-high group demonstrates a lower RNA levels in $M C L$. The gene expression profiles of the EXO.index-high and EXO.index-low groups of patients with MCL are


Figure 4. A total of 10 EXOSC genes were used as classifiers for the 123 patients with MCL. (A) Unsupervised clustering of the expression of 10 EXOSC genes for the 123 patients with MCL. The cluster of EXOSC genes demonstrated cosine correlation similarity. (B) Fuzzy clustering of the 123 patients with MCL based on the expression of the 10 EXOSC genes. (C) Kaplan-Meier curves for overall survival of 123 patients with MCL based on the EXO.index $\left(\mathrm{P}=1.73 \times 10^{-7}\right)$. The log-rank test was used to compare Kaplan-Meier curves. EXOSC, exosome complex; EXO.index, exosome complex index; MCL, mantle cell lymphoma; EXOSC5, exosome component 5; EXOSC1, exosome component 1; EXOSC4, exosome component 4; EXOSC2, exosome component 2; EXOSC7, exosome component 7; EXOSC3, exosome component 3; EXOSC8, exosome component 8; EXOSC6, exosome component 6; EXOSC9, exosome component 9; EXOSC10, exosome component 10 .

demonstrated in Fig. 5A. Overall, it was observed that 153 genes were upregulated and 303 genes were downregulated between the EXO.index-high and EXO. Index-low group 
A
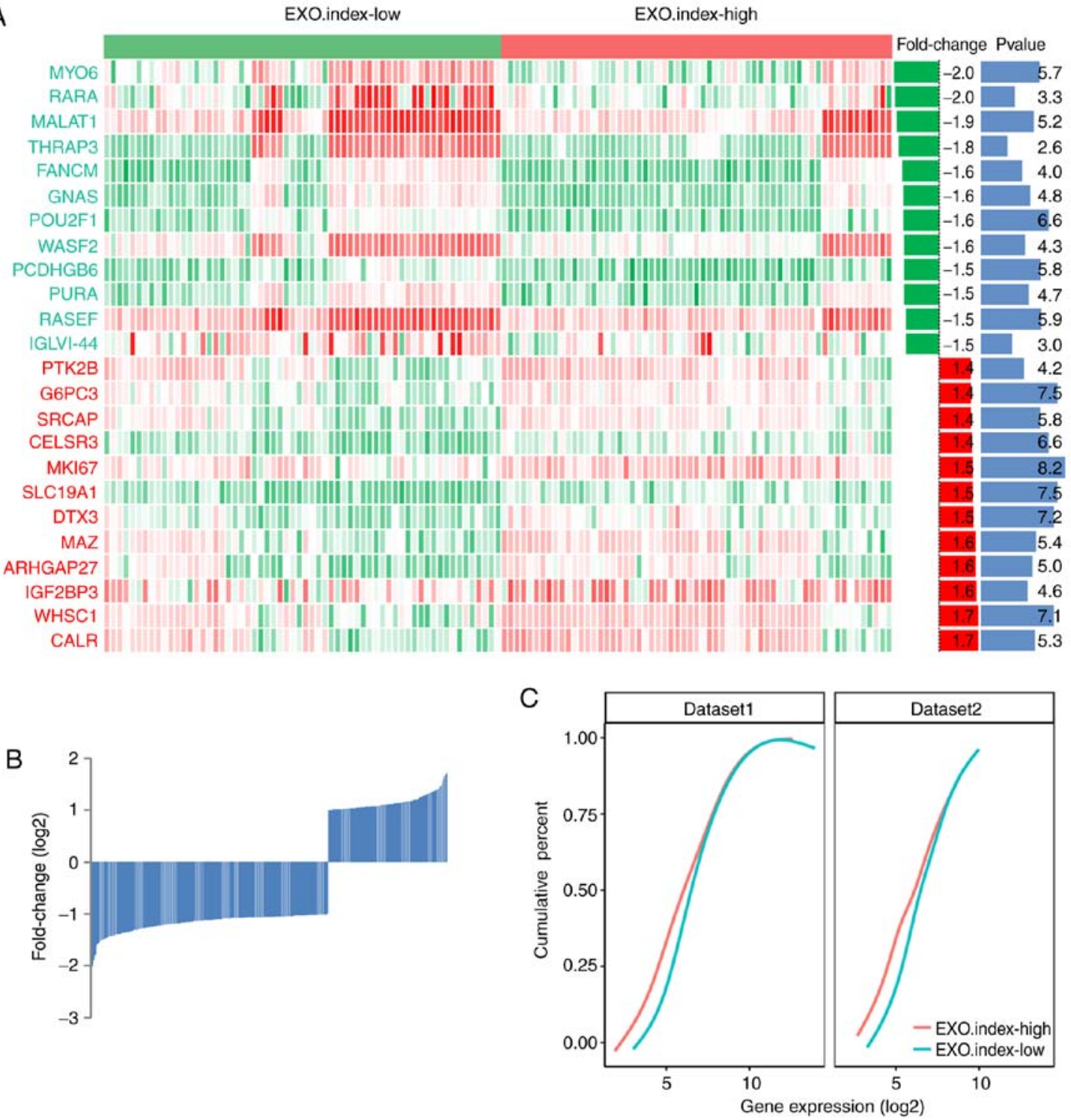

Figure 5. Differentially expressed genes between the EXO.index-high and EXO.index-low groups of 123 patients with MCL. (A) The heatmap demonstrates the differentially expressed genes between the EXO.index-high and EXO.index-low groups. The red color represents high expression, the green color represents low expression and the white color represents intermediate expression. The top 12 downregulated and 12 upregulated genes were included. On the right-hand side of the heatmap, the bar graphs on the left indicate the fold changes $(\log 2)$ and the right bar graph indicates the P-values (-log10). (B) The total of 303 downregulated genes and 153 upregulated genes were identified between the EXO.index-high and EXO.index-low groups of patients with MCL. (C) Cumulative distribution of RNA expression levels of different genes between the EXO.index-high and EXO.index-low groups in patients with MCL (log2 fold change). The left plot represents the GSE93291 dataset $\left(\mathrm{n}=123\right.$ samples; $\left.\mathrm{P}=5.14 \times 10^{-3}\right)$ and the right plot represents the GSE36000 dataset $\left(\mathrm{n}=38 \mathrm{samples} ; \mathrm{P}=9.20 \times 10^{-7}\right)$. EXO.index, exosome complex index; MCL, mantle cell lymphoma.

$(\mathrm{P}<0.05$; Fig. 5B). The number of downregulated genes in the EXO.index-high group of patients with MCL was increased compared with the upregulated genes, indicating that the EXO.index-high group has different ways of RNA processing compared with the EXO.index-low group. As demonstrated in the cumulative distribution of RNA expression of the differentially expressed genes between the EXO.index-high vs. EXO.index-low groups of patients with MCL, the EXO. index-high group exhibited decreased RNA levels compared to the whole transcript profile $\left(\mathrm{P}=5.14 \times 10^{-3}\right.$; Fig. 5C). This result was also confirmed in a second dataset (GSE36000; $\mathrm{n}=38$ samples; $\mathrm{P}=9.20 \times 10^{-7}$; Fig. $5 \mathrm{C}$ ).

Furthermore, the correlation coefficients of the EXO.index with the gene expression of 10 proliferation-associated genes, including $\mathrm{Ki} 67$, were determined in the $123 \mathrm{MCL}$ samples (Fig. S1). The results indicated that EXO.index is highly correlated with the 10 proliferation-associated genes.

$D N A$ repair and regulation of $B$-cell pathways are significantly enriched in MCL. Enrichment analysis of the differentially expressed genes between the EXO.index-high and the EXO. index-low group of patients with MCL was then performed. The results of the GO pathway analysis are summarized in Table I, and the complete list is presented in Table SI. As indicated in Fig. 6A, 'mitotic nuclear division' followed by 'DNA repair' was the most significantly enriched pathway among the cell division-associated pathways, and 'immunoglobulin receptor binding pathway' and 'positive regulation of B-cell 
Table I. GO pathway analysis of differently expressed genes.

\begin{tabular}{llrrr}
\hline Category & \multicolumn{1}{c}{ Term } & Count & P-value & FDR \\
\hline GOTERM_MF_DIRECT & GO:0034987 immunoglobulin receptor binding & 9 & $8.0 \times 10^{-09}$ & $3.7 \times 10^{-06}$ \\
GOTERM_MF_DIRECT & GO:0003823 antigen binding & 11 & $1.1 \times 10^{-05}$ & $4.9 \times 10^{-03}$ \\
GOTERM_MF_DIRECT & GO:0005524 ATP binding & 48 & $3.3 \times 10^{-05}$ & $1.5 \times 10^{-02}$ \\
GOTERM_MF_DIRECT & GO:0005515 protein binding & 182 & $1.6 \times 10^{-04}$ & $7.1 \times 10^{-02}$ \\
GOTERM_MF_DIRECT & GO:0008017 microtubule binding & 13 & $2.5 \times 10^{-04}$ & $1.1 \times 10^{-01}$ \\
GOTERM_MF_DIRECT & GO:0004386 helicase activity & 7 & $3.4 \times 10^{-03}$ & $7.9 \times 10^{-01}$ \\
GOTERM_MF_DIRECT & GO:0003777 microtubule motor activity & 6 & $1.2 \times 10^{-02}$ & $1.0 \times 10^{0}$ \\
GOTERM_MF_DIRECT & GO:0004252 serine-type endopeptidase activity & 11 & $1.3 \times 10^{-02}$ & $1.0 \times 10^{0}$ \\
GOTERM_MF_DIRECT & GO:0003682 chromatin binding & 14 & $1.8 \times 10^{-02}$ & $1.0 \times 10^{0}$ \\
GOTERM_MF_DIRECT & GO:0008253 5'-nucleotidase activity & 3 & $2.3 \times 10^{-02}$ & $1.0 \times 10^{0}$ \\
GOTERM_MF_DIRECT & GO:0003677 DNA binding & 40 & $2.9 \times 10^{-02}$ & $1.0 \times 10^{0}$ \\
GOTERM_MF_DIRECT & GO:0003690 double-stranded DNA binding & 5 & $5.0 \times 10^{-02}$ & $1.0 \times 10^{0}$ \\
GOTERM_MF_DIRECT & GO:0042393 histone binding & 6 & $5.9 \times 10^{-02}$ & $1.0 \times 10^{0}$ \\
GOTERM_MF_DIRECT & GO:0004674 protein serine/threonine kinase activity & 12 & $6.0 \times 10^{-02}$ & $1.0 \times 10^{0}$ \\
GOTERM_MF_DIRECT & GO:0098641 cadherin binding involved in cell-cell adhesion & 10 & $6.2 \times 10^{-02}$ & $1.0 \times 10^{0}$ \\
GOTERM_MF_DIRECT & GO:0035662 Toll-like receptor 4 binding & 2 & $6.7 \times 10^{-02}$ & $1.0 \times 10^{0}$ \\
GOTERM_MF_DIRECT & GO:0051015 actin filament binding & 6 & $7.7 \times 10^{-02}$ & $1.0 \times 10^{0}$ \\
GOTERM_MF_DIRECT & GO:0050544 arachidonic acid binding & 2 & $8.3 \times 10^{-02}$ & $1.0 \times 10^{0}$ \\
GOTERM_MF_DIRECT & GO:0008026 ATP-dependent helicase activity & 3 & $8.8 \times 10^{-02}$ & $1.0 \times 10^{0}$ \\
GOTERM_MF_DIRECT & GO:0004672 protein kinase activity & 11 & $9.0 \times 10^{-02}$ & $1.0 \times 10^{0}$ \\
\hline
\end{tabular}

GO, Gene Ontology; FDR, false discovery rate.

activation' were the 2 most enriched pathways in B-cell immune-associated pathways. Among all of the differentially expressed genes, FANCA and INO80D were upregulated or downregulated in DNA repair pathway (Fig. 6B). According to a previous study, knockdown of EXOSC8 induced cell-cycle exit and promoted the expression of several cell cycle regulatory genes that are involved in cell-cycle arrest (33). A similar expression pattern of these genes was also observed in the other dataset (GSE36000; n=38 samples; Fig. 6B).

\section{Discussion}

In eukaryotic cells, the exosome complex exists in the cytoplasm, nucleus and particularly the nucleolus. In the nucleus, the exosome is required to correct processing of several small RNA molecules, including rRNA, small nuclear (sn) RNA and small nucleolar RNA (34). In the nucleolus, the exosome is involved in the processing of the 5.8S rRNA and several snRNAs (34-36). In the cytoplasm, the exosome has a role in degrading mRNA $(9,37,38)$. It has been demonstrated that mutations in EXOSC3 and EXOSC8 are associated with human neurological diseases $(27,39)$. However, the biological implications and prognostic significance of EXOSC family genes in MCL remain unknown. By analyzing the expression of EXOSC genes in MCL, the present Bioinformatics study indicated that abnormal EXOSC gene expression may predict survival in MCL and alter the expression levels of RNAs compared with those in the whole transcript profile.

MCL is a rare and refractory subtype of non-Hodgkin's lymphomas, with a median overall survival time of 5-7 years.
In previous studies, 20 'proliferation signature' genes (23), a PCR-based 5-gene model (24), the Ki67 proliferation index (25), and the MIPI (26) were used as prognostic models for predicting the survival of patients with MCL. Among these prognostic models, MIPI is the most commonly used, and is applied to stratify patients with MCL into three risk groups: Low risk; intermediate risk; and high risk, on the basis of age, leukocyte count, Eastern Cooperative Oncology Group performance status and lactic dehydrogenase levels $(22,40)$. However, it is not universally accepted for predicting survival in MCL. Therefore, novel biomarkers for predicting the survival of MCL are urgently required (26). In the present analysis, it was indicated that the expression levels of more than one-half of the EXOSC genes (6 out of 10) were significantly associated with survival in MCL $(\mathrm{P}<0.05$; log-rank test). Furthermore, a comprehensive EXO. index was established to predict the survival of patients with MCL. The EXO.index exhibited an improved performance in predicting survival compared with each specific EXOSC protein alone $\left(\mathrm{P}=1.73 \times 10^{-7}\right)$. Furthermore, the unequal expression of EXOSC genes may predict poorer survival in patients with MCL.

In addition, 3 key results from the present study were identified to support the study conclusions. Firstly, the expression of EXOSC genes was demonstrated to be a good classifier in MCL in the fuzzy clustering. Furthermore, the EXO.index was determined, and the patients with MCL were stratified into EXO.index-high and EXO.index-low groups. The EXO. index-high group exhibited decreased RNA levels compared with the whole transcript profile. In addition, the differentially 
A



B


EXO.index-high

EXO.index-low

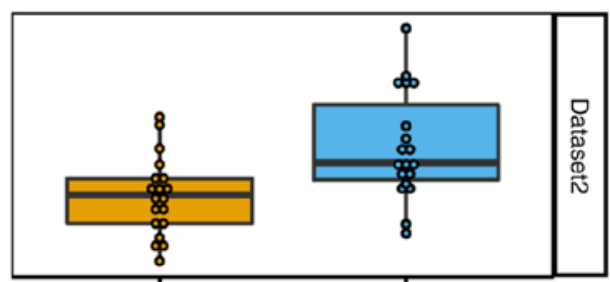

EXO.index-high

EXO.index-low

Figure 6. Significantly enriched pathways between patients with MCL in the EXO.index-high and EXO.index-low groups. (A) Bar graph displaying the distinctly enriched cell division-associated pathways (left-hand side) and B-cell immune-associated pathways (right-hand side). (B) Differentially expressed genes in the DNA repair pathway between EXO.index-high and EXO.index-low groups in patients with MCL. The left plot represents the GSE93291 dataset $(n=123$ samples; $P<0.05)$, and the right plot is for the GSE36000 dataset $(n=38$ samples; $P<0.05)$. EXO.index, exosome complex index; MCL, mantle cell lymphoma; FANCA, FA complementation group A; INO80D, INO80 complex subunit D.

expressed genes in the EXO.index-high group were enriched in cell division and DNA repair pathways, which may lead to poor survival of patients with MCL.

However, there are certain limitations to the present study: There may be confounding factors during the survival comparison of EXO.index-high and EXO.index-low groups for the absent of comparison all the clinical information between each group. As compensation, the correlation coefficient of EXO. index with the gene expression of 10 proliferation-associated genes, including KI67, was calculated in 123 MCL samples. The results indicated that the EXO.index is highly correct with the 10 proliferation-associated genes.
In conclusion, the expression of EXOSC genes was demonstrated to be a good classifier in MCL. Abnormal expression of EXOSC genes predicts poor survival in MCL. Furthermore, the patients with MCL in the EXO.index-high group exhibited poorer survival rates and decreased RNA levels compared with the whole transcript profile. The EXOSC genes were indicated to be associated with cell division and DNA repair pathways, which may result in poorer survival of patients with MCL.

\section{Acknowledgements}

Not applicable. 


\section{Funding}

The present study was financially supported by National Natural Science Foundation of China (grant no. 81800195), the Interdisciplinary Medicine Seed Fund of Peking University (grant no. BMU2018MB004), the Beijing Natural Science Foundation (grant nos. 7132183 and 7182178) and the China Health Promotion Foundation (grant no. CHPF-zlkysx-001).

\section{Availability of data and materials}

The datasets used during the present study are available from the corresponding author upon reasonable request.

\section{Authors' contributions}

HJ and XRL conceived the project. WZ, JZ and XH analyzed the data. WZ, JZ, XH, XNL, JL, WL, PY, JW, $\mathrm{KH}, \mathrm{HMJ}, \mathrm{XRL}$ and $\mathrm{XZ}$ contributed towards the interpretation of the data. All authors wrote and approved the final manuscript.

\section{Ethics approval and consent to participate}

Not applicable.

\section{Patient consent for publication}

Not applicable.

\section{Competing interests}

The authors declare that they have no competing interests.

\section{References}

1. Houseley $\mathrm{J}$ and Tollervey D: The many pathways of RNA degradation. Cell 136: 763-776, 2009.

2. Wilusz CJ, Wormington M and Peltz SW: The cap-to-tail guide to mRNA turnover. Nat Rev Mol Cell Biol 2: 237-246, 2001.

3. Lehner B and Sanderson CM: A Protein Interaction Framework for Human mRNA Degradation. Genome Res 14: 1315-1323, 2004

4. Wang $\mathrm{Z}$ and Kiledjian M: Functional link between the mammalian exosome and mRNA decapping. Cell 107: 751-762, 2001.

5. Chen CY, Gherzi R, Ong SE, Chan EL, Raijmakers R, Pruijn GJ, Stoecklin G, Moroni C, Mann M and Karin M: AU binding proteins recruit the exosome to degrade ARE-containing mRNAs. Cell 107: 451-464, 2001.

6. Schilders G, van Dijk E, Raijmakers R and Pruijn GJ: Cell and molecular biology of the exosome: How to make or break an RNA. Int Rev Cytol 251: 159-208, 2006.

7. van Dijk EL, Schilders G and Pruijn GJ: Human cell growth requires a functional cytoplasmic exosome, which is involved in various mRNA decay pathways. RNA 13: 1027-1035, 2007.

8. Anderson JR, Mukherjee D, Muthukumaraswamy K, Moraes KC, Wilusz CJ and Wilusz J: Sequence-specific RNA binding mediated by the RNase PH domain of components of the exosome. RNA 12: 1810-1816, 2006.

9. Lejeune F, Li X and Maquat LE: Nonsense-mediated mRNA decay in mammalian cells involves decapping, deadenylating, and exonucleolytic activities. Mol Cell 12: 675-687, 2003.

10. Zhang C, Chen Y, Sun B, Wang L, Yang Y, Ma D, Lv J, Heng J, Ding Y, Xue Y, et al: $\mathrm{m}^{6} \mathrm{~A}$ modulates haematopoietic stem and progenitor cell specification. Nature 549: 273-276, 2017.

11. Li HB, Tong J, Zhu S, Batista PJ, Duffy EE, Zhao J, Bailis W, Cao G, Kroehling L, Chen Y, et al: $\mathrm{m}^{6} \mathrm{~A}$ mRNA methylation controls $\mathrm{T}$ cell homeostasis by targeting the IL-7/STAT5/SOCS pathways. Nature 548: 338-342, 2017.
12. Anderson JR, Armitage JO and Weisenburger DD: Epidemiology of the non-Hodgkin's lymphomas: Distributions of the major subtypes differ by geographic locations. Non-hodgkin's lymphoma classification project. Ann Oncol 9: 717-720, 1998.

13. Skarbnik AP and Goy AH: Mantle cell lymphoma: State of the art. Clin Adv Hematol Oncol 13: 44-55, 2015.

14. Zhou Y, Wang H, Fang W, Romaguer JE, Zhang Y, Delasalle KB, Kwak L, Yi Q, Du XL and Wang M: Incidence trends of mantle cell lymphoma in the United States between 1992 and 2004 Cancer 113: 791-798, 2008.

15. Ghielmini M and Zucca E: How I treat mantle cell lymphoma. Blood 114: 1469-1476, 2009.

16. Dreyling $\mathbf{M}$ and Hiddemann W; European MCL Network: Current treatment standards and emerging strategies in mantle cell lymphoma. Hematology Am Soc Hematol Educ Program 542-551, 2009.

17. Zucca E, Stein H and Coiffier B: European lymphoma task force (ELTF): Report of the workshop on mantle cell lymphoma (MCL). Ann Oncol 5: 507-511, 1994.

18. Banks PM, Chan J, Cleary ML, Delsol G, De Wolf-Peeters C, Gatter K, Grogan TM, Harris NL, Isaacson PG, Jaffe ES, et al: Mantle cell lymphoma. A proposal for unification of morphologic, immunologic, and molecular data. Am J Surg Pathol 16: 637-640, 1992.

19. Rajabi B and Sweetenham JW: Mantle cell lymphoma: Observation to transplantation. Ther Adv Hematol 6: 37-48, 2015.

20. Herrmann A, Hoster E, Zwingers T, Brittinger G, Engelhard M, Meusers P, Reiser M, Forstpointner R, Metzner B, Peter N, et al: Improvement of overall survival in advanced stage mantle cell lymphoma. J Clin Oncol 27: 511-518, 2009.

21. Martin P, Chadburn A, Christos P, Furman R, Ruan J, Joyce MA, Fusco E, Glynn P, Elstrom R, Niesvizky R, et al: Intensive treatment strategies may not provide superior outcomes in mantle cell lymphoma: Overall survival exceeding 7 years with standard therapies. Ann Oncol 19: 1327-1330, 2008.

22. Vose JM: Mantle cell lymphoma: 2012 update on diagnosis, risk-stratification, and clinical management. Am J Hematol 87: 604-609, 2012.

23. Rosenwald A, Wright G, Wiestner A, Chan WC, Connors JM, Campo E, Gascoyne RD, Grogan TM, Muller-Hermelink HK, Smeland EB, et al: The proliferation gene expression signature is a quantitative integrator of oncogenic events that predicts survival in mantle cell lymphoma. Cancer Cell 3: 185-197, 2003.

24. Hartmann E: Five-gene model to predict survival in mantle-cell lymphoma using frozen or formalin-fixed, paraffin-embedded tissue. J Clin Oncol 26: 4966-4972, 2008.

25. Determann O,Hoster E, Ott G, Wolfram Bernd H,Loddenkemper C, Leo Hansmann M, Barth TE, Unterhalt M, Hiddemann W, Dreyling M, et al: Ki-67 predicts outcome in advanced-stage mantle cell lymphoma patients treated with anti-CD20 immunochemotherapy: Results from randomized trials of the European MCL network and the German low Grade lymphoma Study Group. Blood 111: 2385-2387, 2008

26. Hoster E, Dreyling M, Klapper W, Gisselbrecht C, van Hoof A, Kluin-Nelemans HC, Pfreundschuh M, Reiser M, Metzner B, Einsele H, et al: A new prognostic index (MIPI) for patients with advanced-stage mantle cell lymphoma. Blood 111: 558-565, 2008.

27. Wan J, Yourshaw M, Mamsa H, Rudnik-Schöneborn S, Menezes MP, Hong JE, Leong DW, Senderek J, Salman MS, Chitayat D, et al: Mutations in the RNA exosome component gene EXOSC3 cause pontocerebellar hypoplasia and spinal motor neuron degeneration. Nat Genet 44: 704-708, 2012.

28. Scott DW, Abrisqueta P, Wright GW, Slack GW, Mottok A, Villa D, Jares P, Rauert-Wunderlich H, Royo C, Clot G, et al: New molecular assay for the proliferation signature in mantle cell lymphoma applicable to formalin-fixed paraffin-embedded biopsies. J Clin Oncol 35: 1668-1677, 2017.

29. Huang da W, Sherman BT and Lempicki RA: Systematic and integrative analysis of large gene lists using DAVID bioinformatics resources. Nat Protoc 4: 44-57, 2009.

30. Ashburner M, Ball CA, Blake JA, Botstein D, Butler H, Cherry JM, Davis AP, Dolinski K, Dwight SS, Eppig JT, et al: Gene ontology: Tool for the unification of biology. The Gene Ontology Consortium. Nat Genet 25: 25-29, 2000.

31. The Gene Ontology Consortium: The Gene Ontology Resource: 20 years and still GOing strong. Nucleic Acids Res 47: D330-D338, 2019.

32. Mi H, Muruganujan A, Ebert D, Huang X and Thomas PD: PANTHER version 14: More genomes, a new PANTHER GO-slim and improvements in enrichment analysis tools. Nucleic Acids Res 47: D419-D426, 2019. 
33. Mciver SC, Kang YA, Devilbiss AW, O'Driscoll CA, Ouellette JN Pope NJ, Camprecios G, Chang CJ, Yang D, Bouhassira EE, et al: The exosome complex establishes a barricade to erythroid maturation. Blood 124: 2285-2297, 2014.

34. Allmang C, Kufel J, Chanfreau G, Mitchell P, Petfalski E and Tollervey D: Functions of the exosome in rRNA, snoRNA and snRNA synthesis. EMBO J 18: 5399-5410, 1999.

35. Mitchell P, Petfalski E, Shevchenko A, Mann M and Tollervey D: The Exosome: A conserved eukaryotic RNA processing complex containing multiple 3' $\rightarrow$ 5'Exoribonucleases. Cell 91: 457-466, 1997.

36. Schilders G, Raijmakers R, Raats JM and Pruijn GJ: MPP6 is an exosome-associated RNA-binding protein involved in $5.8 \mathrm{~S}$ rRNA maturation. Nucleic Acids Res 33: 6795-6804, 2005.

37. Wilson MA, Meaux S and van Hoof A: A genomic screen in yeast reveals novel aspects of nonstop mRNA metabolism. Genetics 177: 773-784, 2016.

38. Lin WJ, Duffy A and Chen CY: Localization of AU-rich element-containing mRNA in cytoplasmic granules containing exosome subunits. J Biol Chem 282: 19958-19968, 2007.
39. Boczonadi V, Müller JS, Pyle A, Munkley J, Dor T, Quartararo J, Ferrero I, Karcagi V, Giunta M, Polvikoski T, et al: EXOSC8 mutations alter mRNA metabolism and cause hypomyelination with spinal muscular atrophy and cerebellar hypoplasia. Nat Commun 5: 4287, 2014.

40. Eskelund CW, Dahl C, Hansen JW, Westman M, Kolstad A, Pedersen LB, Montano-Almendras CP, Husby S, Freiburghaus C, Ek S, et al: TP53 mutations identify younger mantle cell lymphoma patients who do not benefit from intensive chemoimmunotherapy. Blood 130: 1903-1910, 2017.

This work is licensed under a Creative Commons Attribution-NonCommercial-NoDerivatives 4.0 International (CC BY-NC-ND 4.0) License. 